\title{
LOS CENTROS DE INVESTIGACIÓN EN CHIAPAS Y SUS REVISTAS: $1985-2010$
}

\author{
Gabriel Ascencio Franco
}

Resumen: Este trabajo repasa la justificación y objetivos planteados en la fundación de los centros de investigación de ciencias sociales asentados en Chiapas, los contrasta con el énfasis disciplinario, temático, ubicación y escala de 956 artículos publicados en 68 volúmenes de los anuarios y revistas editadas por dichas entidades entre 1985 y 2010, como primer paso para una caracterización de la comunidad académica local.

Palabras clave: Comunidad académica local, institucionalización de las ciencias sociales.

Enviado a dictamen: 07 de junio de 2011

Aprobación: 20 de junio de 2010

Revisiones: 1

Gabriel Ascencio Franco, doctor en Antropología por la Universidad Nacional Autónoma de México. Investigador de Proimmse-IIA-UNAM, Correo electrónico: gafranco@unam.mx.
Abstract: This paper reviews the justification and objectives in the founding of the centers of social science research settled in Chiapas, in contrast to the emphasis on discipline, theme, location and scale of 956 articles published in 68 volumes of yearbooks and magazines published by such institutions between 1985 and 2010, as a first step a characterization of the local academic community.

Keywords: Local academic community, the institutionalization of the social sciences.

\section{Introducción}

L os organizadores del segundo Foro: Las ciencias sociales en Chiapas, celebrado en mayo de 2010 por la Universidad Intercultural de Chiapas en San Cristóbal de Las Casas, me distinguieron con la invitación para presentar una charla acerca de dicho tema. Frente a tal compromiso me di a la tarea de reunir información sobre los centros de investigación asentados en el estado. Pude allegarme algunos informes anuales, comentarios sobre la fundación publicados por sus iniciadores e incluso documentos preparatorios para su creación, además de una colección completa de las revistas de ciencias sociales publicadas en los últimos veinticinco años 
en dichos centros. De estos materiales parten mis observaciones.

Capturar artículo por artículo y ensayar una clasificación útil a la reflexión sobre el desarrollo de las disciplinas sociales en Chiapas me ocupó mucho más tiempo que el par de meses previos a la charla comprometida. Presento ahora el resultado de este trabajo traducido en una decena de gráficos que destacan ciertos rasgos de la comunidad académica chiapaneca y los contrasto con los objetivos perseguidos al crear los centros de investigación. Los índices por revista, autor, lugar y tema de los 956 trabajos publicados entre 1985 y 2010 en 68 volúmenes de las revistas ICACH, CIHMECH, Anuario CEI/IEI, Anuario ICHC/CESMECA, LiminaR, y Pueblos y Fronteras, podrán consultarse en un libro electrónico en línea en la página web: http:// proimmse.unam.mx/. Sin duda serán de utilidad a los interesados en el análisis del desarrollo de las ciencias sociales en el estado y a tesistas e investigadores noveles para ubicar conjuntos de escritos de un tema o autor al inicio de un nuevo trabajo.

Contar con un cúmulo tal de artículos en los últimos veinticinco años es resultado del esfuerzo compartido por los practicantes de las ciencias sociales en Chiapas, en otros lugares de México e incluso en otros países; pero particularmente es resultado del compromiso con el conocimiento que mantienen los integrantes de los centros asentados en San Cristóbal de Las Casas y Tuxtla Gutiérrez, espacios que vinieron a enriquecer y diversificar el panorama de las ciencias sociales y humanísticas de la primera mitad de los ochenta, reducidas en aquel tiempo a las iniciativas del Instituto de Ciencias y Artes de Chiapas. Se trata de:

- El Colegio de la Frontera Sur -ECOSUR-, el cual desde su fundación en 1975 como Centro de Investigaciones Ecológicas del Sureste - CIESno se reduce al cultivo de estas disciplinas.

- El Instituto de Estudios Indígenas - IEI- de la Universidad Autónoma de Chiapas - UNACH- denominado inicialmente Centro de Estudios Indígenas - CEI-, creado formalmente en 1984 aunque sus actividades de investigación arrancaron el siguiente año.

- La Unidad Sureste del Centro de Investigaciones Superiores en Antropología Social -CIESASSureste- surgido en 1985.

- El Centro de Investigaciones Humanísticas de Mesoamérica y el Estado de Chiapas CIHMECH一, nacido en 1985 también y transformado en Programa de Investigaciones Multidisciplinarias sobre Mesoamérica y el Sureste - PROIMMSE - adscrito al Instituto de Investigaciones Antropológicas - IIA- de la Universidad Nacional Autónoma de México -UNAM- en 1998.

- El Centro de Estudios Superiores de México y Centroamérica -CESMECA- perteneciente a la Universidad de Ciencias y Artes de Chiapas, fundado en 1995 como continuación del Departamento de Patrimonio e Investigación creado en diciembre de 1989 por el Instituto Chiapaneco de Cultura - ICHC.

Hoy estos cinco centros reúnen a más de setenta investigadores sociales, se apoyan mutuamente, organizan con frecuencia publicaciones y actividades académicas compartidas, algunos son maestros y asesores en los posgrados de otros, varios fueron o son estudiantes de los mismos. El préstamo interbibliotecario es ágil, se publican libros en coedición y se escribe para las revistas de los otros. El acervo se ha enriquecido notablemente en gran parte por la buena gestión de los directores y bibliotecarios. Todo ello remite a la existencia de una comunidad académica local de ciencias sociales en Chiapas, específicamente en San Cristóbal, de la que también forma parte la Facultad de Ciencias Sociales de la UNACH, la UNICH y la carrera de historia de la UNICACH; pero antes fue diferente. Así se desprende de los documentos de creación de los 
centros de investigación y de algunas de las reflexiones posteriores de sus fundadores.

\section{Los centros de investigación social}

El anteproyecto del Centro de Estudios Indígenas de la UNACH, fechado el dos de marzo de 1984, luego de describir la importancia numérica de la población indígena en Chiapas expone las razones históricas, étnicas, antropológicas y lingüísticas que convocan el interés de su estudio y repasa los pensadores que se han ocupado de dichos temas, desde los conquistadores y misioneros, pasando por los viajeros hasta llegar a los proyectos de universidades extranjeras y el Instituto Nacional Indigenista. Precisa "se han producido importantes documentos de diversa índole que no son asequibles ni a nuestros estudiosos ni a nuestros gobernantes" (UNACH, 1984:5). Lamenta la inexistencia de un centro dedicado a la investigación del mundo indígena y sostiene la necesidad de crearlo pues: ${ }^{1}$

Por su medio obtendría la Universidad los conocimientos adecuados para orientar no solamente a sus alumnos, sino sus planes y programas de estudio y servicio social, lo mismo que su desarrollo futuro.

El Gobierno del estado podrá siempre contar con datos científicos al día en los que sus funcionarios podrán basar y fundamentar adecuadamente sus programas de trabajo para el desarrollo y servicio de las áreas indígenas (UNACH, 1984: 13).

\section{Como objetivo general persigue investigar:}

(...) las culturas indígenas de Chiapas y divulgar, tanto a nivel académico como popular, sus resultados, con el fin de informar y sensibilizar a la comunidad universitaria y a la sociedad en general acerca de los valores de las culturas indígenas de Chiapas (UNACH, 1984: 14).
Para ello se propone partir de un enfoque integral con el concurso de las subdisciplinas antropológicas, ofrecer cursos a profesionistas y estudiantes sobre antropología, historia y lenguas de Chiapas, procurar la concientización sobre los valores y prácticas de los grupos autóctonos mediante conferencias, congresos, exposiciones, etcétera; además formar acervos bibliográficos y documentales sobre las culturas indígenas y fomentar los recursos humanos y materiales para la operación del centro (UNACH, 1984: 14-15). ${ }^{2}$

En la misma tesitura, Cuauhtémoc López Sánchez, fundador de CIHMECH recuerda:

(...) la mayoría de las instituciones que efectuaban o patrocinaban las investigaciones eran extranjeras y las nacionales lo hacían con muchas limitaciones y de manera aislada, con escasos canales de comunicación entre sí, por lo que mucha de la información fue subutilizada o no estaba al alcance de los interesados, ni en el estado de Chiapas, ni en México (...) la mayor parte de la bibliografía chiapaneca se encontraba en bibliotecas y centros de información de Estados Unidos de América y Europa (López, 2002: 559).

De tal manera, entre las principales acciones de descentralización geográfica de la UNAM, el informe del rector Jorge Carpizo del año 1985 reporta la creación del CIHMECH:

El Centro de Investigaciones Humanísticas de Mesoamérica y el Estado de Chiapas comenzó a trabajar en colaboración con diversas instituciones, y su principal objetivo consiste en la reafirmación de la identidad de los habitantes de la zona fronteriza sur y el rescate de valores culturales de esa región mesoamericana (Gaceta UNAM, 27-01-1986: 7).

Para su creación - el 15 de enero en la ciudad de México y el 26 de abril en San Cristóbal- la UNAM 
firmó un "convenio de colaboración" con la UNACH, el ayuntamiento municipal de San Cristóbal y los patronatos Fray Bartolomé de Las Casas y Na Bolom para unir sus esfuerzos en la iniciativa, a la que señalaron cinco objetivos, los tres sustantivos de la actividad universitaria -investigar el área mesoamericana, principalmente Chiapas; capacitar recursos humanos en investigaciones históricas, sociales, antropológicas y estéticas; publicar el resultado de sus estudios-; y dos condiciones para facilitarlos: no tener fines de lucro, además de organizar y estructurar los elementos humanos y materiales para cumplir los objetivos anteriores.

Los objetivos de la creación de CIHMECH pueden resumirse en el interés de conformar una comunidad académica especializada en la región y asentada localmente, conectada con las discusiones nacionales e internacionales, con formación de excelencia y dotadas de acervos documentales y bibliográficos adecuados. Un documento sin firma - escrito probablemente por Ángel Robles, primer secretario académico del Centro, en dos hojas con membrete del Patronato Fray Bartolomé de las Casas, del que era presidente, resume los objetivos del CIHMECH con nitidez y los remite a un área más amplia: "El mencionado convenio tiene por objetivo un mayor conocimiento del sureste de México y sus relaciones con el centro del país y Centroamérica; las aportaciones que sus hombres y la naturaleza dan a la cultura y al engrandecimiento del país".

El veinticinco de mayo de 1998 fue publicado el decreto de creación del Programa de Investigaciones Multidisciplinarias sobre Mesoamérica y el Sureste, previo finiquito del convenio de colaboración que fundó al CIHMECH en 1985 (Gaceta UNAM, 25-05-1998: 8-9). En la agenda de la primera coordinadora, el Proimmse debía jugar a mediano plazo "un papel destacado dentro del estudio y el análisis multidisciplinario de la realidad ecológica, social, política y cultural del sureste de nuestro país y de las relaciones entre esta región y los países centroamericanos" (Gall, 2001: 116). ${ }^{3}$
Andrés Fábregas refiere la situación inicial para la creación de la instancia que dio origen al CESMECA en términos similares a los de Cuauhtémoc López Sánchez - fundador de CIHMECH - y a los del documento de creación del Centro de Estudios Indígenas. Luego de contarnos cómo propuso al gobernador Patrocinio González en 1989 el proyecto de transformación del Instituto Chiapaneco de Cultura y la creación de un departamento de estudios culturales, precisa:
El argumento que convenció al gobernador fue el de que no es posible difundir lo que no se conoce. Además, y así se lo hice ver (...) la antropología hecha por las escuelas de Harvard y Chicago habían implantado no sólo una visión de Chiapas sino también un estilo intelectual de enfocar sus problemas y analizar las características de la sociedad y la cultura. Las ciencias sociales, incluyendo en primer lugar a la antropología, con un planteamiento propio, era inexistente en Chiapas por aquellos años (Fábregas, 200l: 123).

Carlos Gutiérrez Alfonzo, actual director de CESMECA, recuerda que desde su creación, en 1995, recibió el encargo de generar conocimientos acerca de las realidades de los pueblos del sur de México y Centroamérica. ${ }^{4}$ Ofrece la licenciatura en Historia, y maestría y doctorado en Ciencias Sociales y Humanísticas, reconocidos por el Consejo Nacional de Ciencias y Tecnología en el Programa Nacional de Posgrados.

Por su parte la Unidad Sureste del Centro de Investigaciones y Estudios Superiores en Antropología Social, también creación de Andrés Fábregas, inició igualmente "en 1985 con la finalidad de promover los estudios de la frontera sur", nos dice el informe del año 2005 y explica los propósitos de la institución a quince años de su fundación. Destaca en el primer punto su afán por: "Ser una institución líder en el sureste del país y Centroamérica en las áreas de investigación, formación y vinculación de alto nivel en las disciplinas antropológicas" (Rivera, 2005: 2-3). 
El informe reconoce el dominio de investigaciones con independencia mutua aunque con fuertes nexos teóricos entre las líneas en que se inscriben. Dedicado a investigaciones etnohistóricas, antropológicas y lingüísticas: "con especial interés en el sur y sureste de México y una creciente apertura hacia América Central, áreas que comparten una historia en común y problemas similares". En docencia desde 1996 imparte en conjunto con la Unidad Occidente una maestría en Antropología. En el horizonte tiene la promoción de un doctorado que le permitiría establecer lazos más estrechos con el Caribe y Centroamérica. En el mismo documento se celebra la consolidación de una planta de personal administrativo estable y eficiente, pero lamenta y plantea como el mayor problema de la Unidad la volatilidad de sus investigadores. "Por ello se considera necesario estimular a los investigadores, creando mecanismos particulares para motivar su permanencia y atenuar la sensación de aislamiento manifestada por algunos" (CIESAS, 2003: 21, 23, 127, 141).

En resumen, los documentos de fundación y los informes de actividades refrendan el interés de establecer una planta académica en ciencias sociales, servida por departamentos de apoyo, particularmente el bibliotecario y administrativo, con un programa de difusión amplio y un perfil de investigación orientado al conocimiento de Chiapas, el Sureste de México y los países de Centroamérica, a excepción de IEI que se plantea específicamente indagar sobre el mundo indígena.

La conformación de una planta académica estable asentada en el estado de Chiapas se manifiesta en el interés de todos los centros, incluso en el informe de CIESAS de 2003 que lamenta las dificultades para lograrlo. El PROIMMSE ese mismo año no contaba con un solo investigador definitivo. El IEI es la excepción, pues la mayoría de su personal permanece desde los primeros años. Ciertamente los centros universitarios estatales a partir del apoyo federal directo a los cuerpos académicos han mejorado su situación en los últimos tiempos al constituirse en Dependencias de Educación Superior, entidades con investigación y docencia superior y de posgrado pero, en verdad, junto con los de adscripción nacional se han desarrollado desde la incertidumbre y han ido formando y especializando su planta académica poco a poco; con financiamiento siempre medido y en ocasiones con poca infraestructura.

En 2010 los académicos suman más de setenta, con doctorado la mayoría y predominio de los formados como antropólogos, quienes representan la mitad del conjunto sin incluir arqueólogos y lingüistas, que define la especificidad de la comunidad local. Además de los representantes de las disciplinas antropológicas destacan los formados en sociología, economía, historia y salud pública (Cuadro 1).

La conformación de la planta académica inició mediante la contratación de unos pocos investigadores con licenciatura, eso llevó a una segunda etapa caracterizada por su crecimiento en número, el estudio para la obtención de posgrados y la depuración y renovación del grupo, seguida por un tercer momento en el que surgen los programas de posgrado, el mejoramiento de los anuarios y la aparición de revistas dictaminadas con mayor profesionalismo.

La cuarta etapa podría ser, como se plantea en los documentos iniciales, ocuparnos de estudiar el Sureste de México y los países de Centroamérica para alcanzar dichos objetivos originales. Las características de los artículos publicados es congruente con el perfil del personal académico del conjunto de los centros, dado el predominio de los profesionales de la antropología y su vocación por los estudios etnográficos y la escala local, predominan los estudios de tal índole, confinados al entorno chiapaneco y los pueblos indígenas, como específicamente se propuso IEI.

Acerca de los demás propósitos fundacionales puede afirmarse el cumplimiento cabal del conjunto aunque con escasez en algunos rubros; sin embargo, se cuenta con recursos materiales, personal de apoyo y acerbos bibliográficos para las actividades de investigación, y 
es destacable el horizonte de creación y difusión del conocimiento. En este aspecto es importantísimo el elevado número de libros editados, particularmente por CESMECA y PROIMMSE, en el terreno de las publicaciones periódicas se suma IEI, aunque con mucho resulta mayor la cantidad de artículos impresos por el primero (Gráfica 1). En conjunto, el número de artículos observa un crecimiento sostenido a lo largo del período 1985-2010 (Gráfica 2).

\section{Las revistas y la comunidad académica local}

La revista ICACH en su tercera época es la primera que aparece en el horizonte de los últimos veinticinco años con dos números misceláneos publicados en 1987 y 1988 por el Instituto de Ciencias y Artes de Chiapas. Se trata de una revista universitaria de transición en el contexto chiapaneco producida por académicos dedicados a la docencia, preocupados por la difusión de conocimientos nuevos en la que tienen cabida trabajos de investigadores profesionales asentados en la ciudad de México, aunque relacionados con el estado o recién asentados en él, en los nacientes centros de investigación. Así lo muestra la participación en estos dos números de investigadores profesionales como Jan de Vos, Andrés Fábregas, Andrés Medina, Carlos Navarrete, Mario Ruz, Jesús Morales y Víctor Esponda; autores, a excepción del primero, que mantienen continuidad en la entrega de trabajos a las revistas locales hasta el presente. ${ }^{5}$

En los mismos años — 1987 y 1988— vieron la luz los dos primeros anuarios de IEI, continuados en los noventa por cinco más y seis en la siguiente década sumando trece hasta la fecha. La revista CIHMECH entre 1988 y 1996 editó seis números con periodicidad irregular. A partir de 1991 se publican los anuarios de ICHC, hoy CESMECA, llegando a veinte volúmenes editados regularmente, acaso con algún retraso.

Particularmente estos anuarios son importantes para comprender el surgimiento de la comunidad académica en Chiapas. En el Departamento de Patrimonio Cultural, con Jesús Morales como director, se constituyó un equipo de investigadores locales, nos recuerda Fábregas y celebra la publicación del Anuario 1990, prácticamente el mismo año que iniciaron las investigaciones. Advierte "la constitución de una nueva generación de científicos sociales y el énfasis en la etnología y la etnohistoria como especializaciones características de la misma" (Fábregas, 2001: 124).

Al comentar el Anuario 1991 Fábregas destaca la producción de conocimientos por los investigadores asentados en las instituciones chiapanecas como fundamentales para entender la coyuntura de 1994:

Debe notarse la capacidad de los investigadores locales en proponer una reflexión realmente importante no sólo en el contexto local, sino también en el nacional y el latinoamericano. En el contexto actual, nadie que desee comprender los acontecimientos de 1994 a nuestros días, puede prescindir de la lectura de este Anuario de 1991 del ICHC. Tal es su importancia. No sobra comentar que por vez primera en muchos años, un grupo de investigadores locales escribía antes que los antropólogos norteamericanos sobre aspectos de la realidad de Chiapas que fueron de su monopolio o en los que jamás repararon. El hecho es importante visto en perspectiva: señala un momento de consolidación y madurez de los investigadores locales en un territorio que solía ser campo exclusivo de los programas de posgrado de universidades como Harvard y Chicago (Fábregas, 2001: 126). ${ }^{6}$

Con referencia al Anuario de IEI, afirma que desde el primero está presente la participación de investigadores que se especializarían en el estudio de los zoques. Agrega que:

Todos estos trabajos mostraban la puesta en marcha de un proceso que fue madurando hasta llegar a nuestros días: la forja de una comunidad académica 
de ciencias sociales, digna de ese nombre, en Chiapas. Todos los que vivimos ese proceso sabemos el alto costo que ello ha tenido y que, quizá, corresponderá a otros y en otro tiempo analizar (Fábregas, 2001: 139-140).

Sobre esas dificultades, el propio Fábregas pone como ejemplo la modestia de la edición del segundo Anuario del IEI y el esfuerzo desplegado por los investigadores para la realización del proyecto común emprendido por todos los académicos para hacer el diagnostico socioeconómico de la población que circunda la reserva ecológica de El Triunfo a la que se dedica todo el volumen. Y agrega que dicho Anuario resulta "signo (...) de las insólitas miserias que rodeaban a la investigación científica en Chiapas". Además, sostiene que dicho trabajo "sigue desempeñando un papel ejemplar y allí está para quien se interese en algún momento en escribir la historia de las ciencias sociales en Chiapas" (Fábregas, 2001: 140).

Las propias investigadoras de IEI consideran que el Anuario de Estudios Indígenas representa una contribución destacada. En un estudio próximo a su publicación dicen:

El primer número del Anuario apareció en 1986 y fue una de las primeras revistas de corte social y antropológico en Chiapas; aún hoy existen pocas publicaciones de este género en la entidad. Originalmente fue concebido para difundir y debatir los avances de los investigadores del recién creado Centro de Estudios Indígenas, ahora Instituto. Abría también espacios para otros estudiosos sobre la cultura, historia y problemática socioeconómica de los grupos humanos asentados en Chiapas y en general el área maya. Los primeros nueve volúmenes responden a esa intención. En los últimos años se ha rediseñado su formato para convertirlo en una revista con una sección temática que permita a los distintos grupos colegiados de la dependencia profundizar en el debate de alguna problemática o algún enfoque específico con colegas invitados (Garza y Fernández, en prensa).

Pero también se quejan de la falta de recursos, del colofón se colige que a veinticinco años de iniciado el trabajo académico en IEI, dicen las autoras:
El Anuario ha sido durante todos estos años nuestro resultado colectivo más importante. Mantenerlo ha significado un enorme esfuerzo, pues los recursos han sido muy escasos y los apoyos mínimos. Todo el trabajo ha recaído en el comité editorial del Instituto, constituido por los mismos académicos. No se ha contado generalmente con el soporte técnico para la corrección de estilo, el diseño, u otras necesidades de edición. Tampoco ha podido el IEI, dadas las limitaciones presupuestales, aceptar trabajos en lenguas distintas al español, salvo muy contadas excepciones, pues el trabajo de traducción rebasa nuestras posibilidades. La edición del Anuario no ha tenido la periodicidad debida; algunos años ha sido necesario posponer la impresión, de modo que solamente han visto la luz, con el actual, catorce volúmenes. A pesar de todo, el esfuerzo editorial del IEI no se ha interrumpido y el Anuario ha logrado posicionarse dignamente entre las publicaciones arbitradas de Chiapas (Garza y Fernández, en prensa).

Andrés Fábregas, luego de comentar los trabajos de la revista CIHMECH y de los anuarios de IEI y CESMECA, refiere:

No puedo evitar el pensar en la importancia que ha revestido el que se consolidara una comunidad académica, un núcleo de pensadores en el estado de Chiapas. En muchos sentidos, la historia de la conformación, maduración, desarrollo y consolidación de un tal grupo, se contiene en las páginas de los anuarios y la revista CIHMECH. No digo que es un 
grupo homogéneo y con el mismo corte ideológico y académico. Bien mirados, los trabajos revisados transmiten una pluralidad de formaciones y puntos de vista que constituye la mejor virtud de este núcleo. Hago votos porque encuentren siempre el camino de revitalizarse, de estar cerca de los más jóvenes y de abrirles el paso en el momento preciso (Fábregas, 2001: 150).

Ejemplo de revitalización y apertura a las nuevas generaciones, con el fin de colocar a CESMECA como una referencia obligada, refiere su director que además del Anuario se publica regularmente la revista semestral LiminaR. Estudios sociales y humanísticos desde el año 2003, misma que ha logrado ubicarse en el interés de los investigadores sociales en el ámbito nacional y en el extranjero por la calidad de sus materiales y por la pertinencia de sus temáticas. Como producto del rigor con la que se edita, ha sido incluida en índices nacionales e internacionales. ${ }^{7}$ Es el caso también de la revista semestral Pueblos y fronteras, de PROIMMSEIIA, publicada en versión impresa los años 2001-2002 y en formato digital a partir de 2006, reemplazando a la revista CIHMECH de aparición irregular. ${ }^{8}$

El número de contribuciones publicadas anualmente en las revistas de ciencias sociales de Chiapas a finales de los ochenta fue duplicado en los años noventa; y vuelto a duplicar en la siguiente década (Gráfica 2). Predominan, relacionados con la formación disciplinaria de los investigadores que constituyen el grupo, los trabajos referidos a temas antropológicos, seguidos de los históricos, sociológicos y económicos (Gráficas 3 y 4). En los antropológicos destacan identidad étnica y etnografías de diferente énfasis, se echa de menos trabajos sobre lenguas amerindias, estructura agraria y movimiento campesino, dado lo importante del fenómeno en el estado. En cambio, resaltan los trabajos sobre salud, religión y sistemas de producción (Gráfica 5).

De la misma forma, la orientación antropológica de la comunidad explica que la mitad de los estudios publicados son resultados de investigaciones a escala local, municipal o regional (Gráfica 6). En parte, por la misma razón más de sesenta por ciento remiten al contexto de Chiapas dada la abundancia de pueblos indígenas en el estado, pero a la vez tiene que ver con la falta de recursos financieros para trabajo de campo y archivo prolongado fuera del lugar de residencia de los académicos; se contrató profesionistas en proceso de formación y por lo mismo con pocas incursiones - menos de diez por ciento - en estudios de alcance universal, centrados en reflexiones teóricas y generalizaciones. De hecho, muchos de los trabajos de esta naturaleza son de la pluma de intelectuales invitados, al igual que los referidos a Centroamérica, los estados del Sureste de México y del resto del país: veinte por ciento en conjunto (Gráfica 7). Pero al mismo tiempo tiene que ver con la idea de que estar en el estado es para estudiar al propio Chiapas, con mayor razón si el empleador es una institución chiapaneca: los estudios panorámicos, las comparaciones entre diferentes lugares se hacen en el centro, desde el centro.

El número de artículos según la ubicación de las investigaciones y la adscripción de los autores dibujan gráficas muy parecidas. Es decir, los académicos estudian los lugares en donde residen. La cantidad de trabajos publicados acerca de Chiapas es similar a la de los autores adscritos a una entidad de investigación asentada en Tuxtla Gutiérrez o San Cristóbal de Las Casas, y la mayoría de los autores publican en revistas de la propia casa; esto es, en medios editados por su centro de trabajo ubicado en Chiapas, en una u otra de las ciudades referidas. Si se resta el número de investigadores residentes fuera de Chiapas con los trabajos relativos a otras latitudes queda claro que los autores chiapanecos escasamente escriben sobre otros lugares (Gráficas 7 y 8 ).

De las contribuciones $85 \%$ son de un solo autor a pesar del impulso que se viene dando en los últimos años a escala nacional al trabajo en equipos y la publicación en coautoría (Gráfica 9), eso habla de un 
proceso de investigación muy individualista, cuya productividad es evaluada y premiada con becas y sobresueldos que llegan a representar mucho más de la mitad de los ingresos totales de un investigador. En ese derrotero, la comunidad académica chiapaneca cuenta con una docena de autores muy prolíficos, con diez o más artículos publicados solo en sus revistas y casi dos docenas con cinco a nueve. Podría decirse que en este grupo, formado por menos de la mitad de los investigadores locales, son el motor y en él descansa el carácter de la producción académica local (Gráfica 10).

Sin embargo falta valorar el individualismo en los procesos de investigación y autoría. Sabemos que la evaluación y los ingresos se establecen individualmente pero tenemos un buen grupo de libros colectivos y algunas investigaciones de equipo: analizar las condiciones de su producción y la calidad de sus resultados nos orientaría en caminos posibles. Libros como Los rumbos de otra historia, coordinado por Viqueira y Ruz; La tierra en Chiapas, escrito por un equipo encabezado por Villafuerte; Chiapas: la paz en la guerra, compilado por Miranda y Espinosa; La comunidad a debate, coordinado por Lisbona; los libros sobre la remunicipalización en Chiapas, encabezados por Burguete y Leyva, y el propio Anuario 2 de CEII, son ejemplos contrastantes por sus resultados y desarrollo del proceso de investigación. El matiz está expresado en los vocablos coordinado, encabezado, compilado, términos que finalmente remiten a una comunidad académica.

Una reflexión sobre las experiencias de este tipo de libros e investigaciones nos llevaría a enriquecer nuestra perspectiva sobre el trabajo individual y colectivo, interdisciplinario y multidisciplinario, tanto como acerca de ciertos tintes de canibalismo y simulación presentes en la academia nacional que comienzan a manifestarse entre nosotros - acaparamiento de tesistas, intentos de inscripción de media docena de ponencias con un autor común en un solo congreso, demanda de coautoría con tesistas, coautoría simulada en cuerpos académicos, edición de libros colectivos sin unidad-, sin duda relacionados con los sistemas de evaluación, promoción y asignación de recursos para la investigación y salarios.

También nos enriquecería una reflexión detenida sobre las características de los anuarios y revistas en diálogo con las exigencias de dictaminación externa y "académicamente rigurosa" que se procura establecer desde los centros que dictan las normas en el campo de la investigación científica. Una evaluación de tal naturaleza valoraría sin duda la eficacia de tales criterios en el desarrollo y consolidación de una comunidad académica local como la chiapaneca, así como su contribución al desarrollo del conocimiento y su alcance, ya sea local o universal.

\section{Colofón}

En las revistas y anuarios escribimos principalmente los de la propia casa y los colegas de otros centros de San Cristóbal y Tuxtla, son sin duda un espacio de reproducción del grupo, como lo son los programas de posgrado y las reuniones académicas que organizamos y las redes en que participamos; pero también de otros lugares de México, de España, Estados Unidos y otros países europeos, escriben en nuestros medios, eso muestra nuestras redes, no estamos aislados. Sin embargo, muy pocos textos se editan acerca del Sureste y Centroamérica, y quien los escribe son académicos de aquellas latitudes, apenas un puño de los investigadores locales se ha interesado en universos fuera de Chiapas.

Pocos son los trabajos que evalúan un conjunto de textos o se aventuran a la reflexión teórica. Abundan los estudios de caso a escala local, municipal o regional, de alguna manera atados al predominio de la antropología. Escasísimos son los que rebasan el ámbito estatal y muchos menos los que se ocupan de la comparación interestatal o internacional. Es decir, investigamos y escribimos casi solo acerca de Chiapas —somos chiapanecólogos-, debemos preguntarnos 
si falta una visión más amplia que nos ayude a saltar los límites estatales o si acotarse a la entidad resulta útil y es suficiente para la reproducción universitaria local y poner conocimientos adecuados a disposición de los tomadores de decisiones en el Estado, como se planea en el documento preparatorio para la fundación de IEI. Entonces, tendríamos que proponer cambiar los nombres de nuestros centros, aunque también podemos asumir que las denominaciones fueron puestas únicamente para estimular la imaginación de los políticos que tenían que aprobar los proyectos de creación respectivos. Así no haría falta reparar en los ámbitos a que remiten. En los nombres se enfatiza Sureste, Centroamérica y Mesoamérica: CIES/ECOSUR, CIHMECH/PROIMMSE, CESMECA, CIESAS-Sureste.

Hace falta afinar temáticas y perspectivas de investigación para apuntalar un sugerente desarrollo de nuestras investigaciones. Al final, permanece la pregunta acerca del tipo de institucionalización de la investigación que vivimos: zexigencias de utilidad inmediata del conocimiento, premios y logros individualizados, discursos colectivistas y frenos a la investigación en grupo o allende los límites de Chiapas?

\section{Notas}

1 El rector de aquella época recuerda que una delegación del departamento de Lenguas le externó su preocupación por la ausencia de enseñanza de idiomas indígenas. Organizó una reunión para hablar del tema, entre los asistentes se contó con la participación de Tomas Lee y Dolores Aramoni. Fue una lluvia de ideas de la que surgió el CEI con Mario Humberto Ruz como director y un grupo de investigadores en que estaban Raúl Perezgrovas, María Elena Fernández y Dolores Aramoni (Morales, 2005: 16-17).

2 El informe de labores 1996-2000, a quince años de su fundación IEI mantenía la orientación de estudiar preferentemente a los grupos asentados en Chiapas, aunque disminuye el énfasis antropológico e indígena.
También conservaba la divulgación del conocimiento, la organización de reuniones académicas, la formación de investigadores y la consolidación de la información bibliográfica como sus cometidos (Fernández, 2000: 2-3). En 2009, el informe de su directora reporta trece investigadores y tres técnicos. Tres miembros de SNI, tres con grado de doctor, siete inscritos en programas de doctorado, tres más con maestría, una licenciada y dos pasantes de licenciatura, organizados en cuatro cuerpos académicos: Antropología del poder, Organización social e historia, Estudios urbanos, movimientos de población y cambio cultural, y Sistemas de vida y estrategias de desarrollo (Rodríguez, 2009).

${ }^{3}$ Para 2010 cuenta con catorce académicos de tiempo completo: dos técnicos y doce investigadores, trece con estudios doctorales, diez con el grado de doctor alcanzado, cinco incorporados al Sistema Nacional de Investigadores y dos al Sistema Estatal de Investigadores.

${ }^{4}$ El Centro está formado por veintitrés investigadores, de los cuales, catorce tienen el "perfil deseable" establecido por PROMEP. Además, cincuenta por ciento de dichos investigadores pertenece al Sistema Nacional de Investigadores. El Centro ha organizado su labor de investigación a través de tres cuerpos académicos. El primero que tiene por nombre Política, diferencia y fronteras se enfoca al estudio de la política, lo político, el desarrollo, las migraciones y el género, desde la dimensión fronteriza; por sus resultados conjuntos, en 2008 ascendió al grado de Cuerpo académico consolidado, la máxima distinción que otorga el Programa de Mejoramiento del Profesorado, PROMEP, un logro que debe ponderarse en todas sus dimensiones: se trata del primer grupo de investigación de la Universidad que alcanza ese rango. El segundo se denomina Patrimonio sociocultural, y tiene la tarea de estudiar el patrimonio y la historia de esta región de México y Centroamérica, mismo que ha sido reconocido como un cuerpo académico en consolidación. El tercero recibe el nombre de Sociedad y cultura en fronteras, y 
se enfoca al estudio de las dimensiones culturales de las fronteras y las migraciones; es también un cuerpo académico en consolidación.

${ }^{5}$ Con siete años de por medio, al transformarse el Instituto en Universidad de Ciencias y Artes salieron en una nueva época cuatro números más los años 1995 a 1998.

${ }^{6}$ Este texto tiene origen en la participación del Dr. Fábregas en un foro sobre Las revistas de ciencias sociales en Chiapas, convocado por PROIMMSE el año 2000, entre otros cometidos para moderar los criterios que habrían de conducir a la creación de Pueblos y fronteras en sustitución de la revista CIHMECH.

${ }^{7}$ Está en la Red de Revistas Científicas de América Latina y El Caribe, España y Portugal —REDALyC-; en el Sistema regional de información en línea para revistas científicas de América Latina, el Caribe, España y Portugal - LATINDEX - ; en Citas Latinoamericanas en Ciencias Sociales y Humanidades -CLASE-; en el Hispanic American Periodicals Index - HAPI-, promovido por la Universidad de California. Resulta pertinente señalar que en ese año fue la única revista mexicana admitida en dicho índice. Recibió mención especial en el ler. Concurso "Fondo de Apoyo a las revistas de Ciencias Sociales de América Latina y el Caribe Juan Carlos Portantiero", organizado por el Consejo Latinoamericano de Ciencias Sociales CLACSO - con sede en Buenos Aires, Argentina.

${ }^{8}$ Está incluida en el Catálogo de revistas electrónicas del Subsistema de Humanidades de la Universidad Nacional Autónoma de México -http://www. catalogoderevistas.unam.mx/interiores/r/rev_pyf. html—; en la Red de Revistas Científicas de América Latina y el Caribe, España y Portugal -http:// redalyc.uaemex.mx-; en el Améric@nistes Le blog de I'IdA Sud-ouest -http://america.hypotheses.org/ category-; en el Directory of Open Access Journals —http://www.doaj.org/doaj?func=openurl\&genre=jo urnal\&eissn=18704115-; y en el Sistema Regional de Información para las Revistas Científicas de América
Latina, El Caribe, España y Portugal (LATINDEX) http://www.latindex.unam.mx-.

\section{Bibliografía}

CIESAS (2003), Historia del CIESAS y plan de desarrollo institucional. 2003-2010, México: Centro de Investigaciones y Estudios Superiores en Antropología Social.

CIHMECH (1994), Programa académico 1994, sd., México: Universidad Nacional Autónoma de México.

CIHMECH (1996), "Lineamientos de desarrollo institucional a mediano plazo del Centro de Investigaciones Humanísticas de Mesoamérica y el estado de Chiapas", Borrador en archivo electrónico.

Fábregas Puig, Andrés (2001), "Diez años de antropología ehistoria de Chiapas: una presentación de los anuarios del Cesmeca y del IEI y la revista CIHMECH", en Inventario Antropológico. Anuario de la revista Alteridades, vol. 7, Universidad Autónoma Metropolitana, pp. 123-152.

Fernández Galán, María Elena (2000), "Instituto de Estudios Indígenas”, en Informe de labores: 1996-2000, San Cristóbal de Las Casas, México: Universidad Autónoma de Chiapas.

Gall Sonabend, Olivia (2001), Proimmse. Informe global: junio 1998-enero 2000, México: Universidad Nacional Autónoma de México.

Garza Caligaris, Anna María y María Elena Fernández Galán, en prensa, "El Anuario de Estudios Indígenas 1986-2008", Inventario Antropológico. Anuario de la revista Alteridades, Universidad Autónoma Metropolitana.

Lisbona Guillén, Miguel (2004), Proimmse. Plan de desarrollo académico, San Cristóbal de Las Casas, México: Universidad Nacional Autónoma de México. Lisbona Guillén, Miguel (2005), Proimmse. Informe de actividades: septiembre 2004-agosto 2005, San Cristóbal de Las Casas, México: Universidad Nacional Autónoma de México. 
Lisbona Guillén, Miguel (2006), Proimmse Informe de actividades: septiembre 2005-agosto 2006, San Cristóbal de Las Casas, México: Universidad Nacional Autónoma de México.

Lisbona Guillén, Miguel (2007), Proimmse. Informe de actividades: septiembre 2006-agosto 2007, San Cristóbal de Las Casas, México: Universidad Nacional Autónoma de México.

Lisbona Guillén, Miguel (2008), Proimmse. Informe de actividades: septiembre 2007-agosto 2008, San Cristóbal de Las Casas, México: Universidad Nacional Autónoma de México.

Lisbona Guillén, Miguel (2009), Proimmse. Informe de actividades: septiembre 2008-agosto 2009, San Cristóbal de Las Casas, México: Universidad Nacional Autónoma de México.

Lisbona Guillén, Miguel (2010), Proimmse. Informe de actividades: septiembre 2009-agosto 2010, San Cristóbal de Las Casas, México: Universidad Nacional Autónoma de México.

López Sánchez, Cuauhtémoc (2002), "La creación del CIHMECH. Fundación e Historia de los Centros de Investigación", Anuario 2000, Tuxtla Gutiérrez, México: CESMECA-UNICACH, pp. 559-566.

Póhlenz, Juan, Olivia Gall, Mauricio Ortega, Susana Ekholm, Felipe Catalán y Manuel Hidalgo (1997), "Propuesta preliminar para la creación del Centro Regional de Investigaciones Interdisciplinarias del Sureste de México", San Cristóbal de Las Casas:
Universidad Nacional Autónoma de México, mecanuscrito.

Proimmse (1998), "Plan de desarrollo institucional: 1999-2000”, San Cristóbal de Las Casas: Universidad Nacional Autónoma de México, mecanuscrito.

Rodríguez Galván, María Guadalupe (2009), Instituto de Estudios Indígenas. Gestión 2008-2012. Primer informe de actividades, San Cristóbal de Las Casas, México: Universidad Autónoma de Chiapas, archivo electrónico.

Schumann Gálvez, Otto (2001), Proimmse. Informe de actividades: enero 2000-enero 2001, San Cristóbal de Las Casas, México: Universidad Nacional Autónoma de México.

Schumann Gálvez, Otto (2002), Proimmse. Informe de actividades: enero 2001-enero 2002, San Cristóbal de Las Casas, México: Universidad Nacional Autónoma de México.

Schumann Gálvez, Otto (2003), Proimmse. Informe de actividades: enero 2002-enero 2003, San Cristóbal de Las Casas, México: Universidad Nacional Autónoma de México, mecanuscrito.

Unach (1984), "Anteproyecto del Centro de Estudios Indígenas”, Tuxtla Gutiérrez, México: Universidad Autónoma de Chiapas.

UNAM (1998), Acuerdo de creación del "Programa de Investigaciones Multidisciplinarias sobre Mesoamérica y el Sureste", Gaceta UNAM, México: Universidad Nacional Autónoma de México. 
Cuadro 1

Académicos adscritos a los centros de investigación social en Chiapas: 2010

\begin{tabular}{|c|c|c|c|}
\hline INSTITUCIÓN E INVESTIGADOR & $\begin{array}{c}\text { TEMA DE } \\
\text { INVESTIGACIÓN* }\end{array}$ & GRADO ACADÉMICO & DISCIPLINA \\
\hline \multicolumn{4}{|c|}{ CIESAS-Sureste } \\
\hline Araceli Burguete Cal y Mayor & Política & $\mathrm{M}$ & Antropología \\
\hline Carolina Rivera Farfán & Migración & $\mathrm{D}$ & Antropología \\
\hline "Diana Laura Reartes Peñafiel & Salud pública & $\mathrm{D}$ & Antropología \\
\hline Edith Francoise Kauffer Michel & Riesgo & D & Ecología \\
\hline Eladio Mateo Toledo & Lenguas indígenas & D & Lingüística \\
\hline Enrique Eroza Solana & Salud pública & D & Antropología \\
\hline Gabriela Robledo Hernández & Migración & $\mathrm{D}$ & Antropología \\
\hline Gilles Polian Marcus & Lenguas indígenas & $\mathrm{D}$ & Lingüística \\
\hline Graciela Freyernuth Enciso & Salud pública & $\mathrm{D}$ & Antropología \\
\hline Jan De Vos Van Gerven † & Historia & $\mathrm{D}$ & Historia \\
\hline José Luis Escalona Victoria & Política & $\mathrm{D}$ & Antropología \\
\hline María Dolores Palomo & Historia & $\mathrm{D}$ & Historia \\
\hline "María Elena Martínez Torres & S. Producción & D D & Ecología \\
\hline Roberto Zavala Maldonado & Lenguas indígenas & $\mathrm{D}$ & Lingüística \\
\hline Ronald Nigh Nielsen & S. Producción & $\mathrm{D}$ & Antropología \\
\hline Xochitl Leyva Solano & Política & $\mathrm{D}$ & Antropología \\
\hline \multicolumn{4}{|c|}{ ECOSUR } \\
\hline Antonio López Meza & Desarrollo & M & Sociología \\
\hline Antonio Saldívar Moreno & Desarrollo & $\mathrm{D}$ & Educación \\
\hline Ernesto Benito Salvatierra Izaba & Salud pública & $\mathrm{D}$ & Salud pública \\
\hline Esperanza Tuñón Pablos & Demografía & $\mathrm{D}$ & Sociología \\
\hline Fernando Limón Aguirre & Educación & $\mathrm{D}$ & Sociología \\
\hline Georgina Sánchez Ramírez & Género & $\mathrm{D}$ & Salud pública \\
\hline Guillermo Montoya Gómez & Desarrollo & M & Economía \\
\hline
\end{tabular}




\begin{tabular}{||l||l||c||l|}
\hline \hline \multicolumn{1}{|c||}{ INSTITUCIÓN E INVESTIGADOR } & \multicolumn{1}{c||}{$\begin{array}{c}\text { TEMA DE } \\
\text { INVESTIGACIÓN* }\end{array}$} & GRADO ACADÉMICO & DISCIPLINA \\
\hline \hline Héctor Javier Sánchez Pérez & Violencia & D & Salud pública \\
\hline \hline Héctor Ochoa Díaz-López & Salud pública & D & Salud pública \\
\hline \hline Jorge Luis Cruz Burguete & Migración & D & Sociología \\
\hline \hline Manuel Roberto Parra Vázquez & Desarrollo & D & Economía \\
\hline \hline Martha Luz Rojas Wiesner & Migración & D & Sociología \\
\hline \hline R. Germán Martínez Velasco & Migración & D & Antropología \\
\hline \hline Ramón Mariaca Méndez & S. Producción & D & Áńa \\
\hline \hline
\end{tabular}

CESMECA-UNICACH

\begin{tabular}{|c|c|c|c|}
\hline Alain Basail Rodríguez & Migración & $\mathrm{D}$ & Sociología \\
\hline Astrid Maribel Pinto Durán & Identidad & $\mathrm{M}$ & Antropología \\
\hline Axel Michael Köler & Identidad & $\mathrm{D}$ & Antropología \\
\hline Carlos Uriel del Carpio Penagos & Ecología & $\mathrm{D}$ & Antropología \\
\hline Daniel Villafuerte Solís & Migración & $\mathrm{D}$ & Economía \\
\hline Efraín Ascencio Cedillo & Identidad & $\mathrm{L}$ & Antropología \\
\hline Jesús Morales Bermúdez & Literatura & $\mathrm{D}$ & Antropología \\
\hline Jesús Solís Cruz & Política & $\mathrm{D}$ & Antropología \\
\hline Leocadio Edgar Sulca Báez & Identidad & $\mathrm{D}$ & Antropología \\
\hline María del Carmen García Aguilar & Migración & $\mathrm{D}$ & Sociología \\
\hline María del Rocío Ortiz Herrera & Historia & $\mathrm{D}$ & Historia \\
\hline María Inés Castro Apreza & Género & $\mathrm{D}$ & Antropología \\
\hline María Luisa de la Garza Chávez & Identidad & $\mathrm{D}$ & Filosofía \\
\hline Martín de la Cruz López Moya & Identidad & $\mathrm{M}$ & Antropología \\
\hline Mercedes Olivera Bustamante & Género & $\mathrm{D}$ & Antropología \\
\hline Sergio Nicolás Gutiérrez Cruz & Historia & $\mathrm{D}$ & Historia \\
\hline Teresa Ramos Maza & Género & $\mathrm{D}$ & Antropología \\
\hline Tomás A. Lee Whiting & Arqueología & $\mathrm{M}$ & Arqueología \\
\hline Víctor Manuel Esponda Jimeno & Identidad & $\mathrm{M}$ & Antropología \\
\hline
\end{tabular}




\begin{tabular}{|c|c|c|c|}
\hline INSTITUCIÓN E INVESTIGADOR & $\begin{array}{c}\text { TEMA DE } \\
\text { INVESTIGACIÓN* }\end{array}$ & GRADO ACADÉMICO & DISCIPLINA \\
\hline \multicolumn{4}{|c|}{ IEI-UNACH } \\
\hline Anna Ma. Garza Caligaris & Historia & $\mathrm{M}$ & Antropología \\
\hline Antonio Gómez Hernández & Etnicidad & M & Antropología \\
\hline Dolores Aramoni Calderón & Etnohistoria & $\mathrm{M}$ & Antropología \\
\hline Gracia Imberton Deneke & Etnicidad & $\mathrm{M}$ & Antropología \\
\hline Jorge Gustavo Paniagua Mijangos & Identidad & M & Antropología \\
\hline Jorge Ignacio Angulo Barredo & Migración & M & Antropología \\
\hline Laureano Reyes Gómez & Etnicidad & $\mathrm{D}$ & Antropología \\
\hline Ma. de Lourdes Zaragoza Martínez & Etnoveterinaria & M & Veterinaria \\
\hline Ma. Elena Fernández-Galán & Lenguas indígenas & M & Lingüística \\
\hline Ma. Guadalupe Rodríguez Galván & Etnoveterinaria & $\mathrm{M}$ & Veterinaria \\
\hline Raúl Perezgrovas Garza & Etnoveterinaria & $\mathrm{D}$ & Veterinaria \\
\hline Sonia Toledo Tello & Sociedad rural & $\mathrm{M}$ & Antropología \\
\hline Susana Villasana Benítez & Demografía & $\mathrm{D}$ & Antropología \\
\hline \multicolumn{4}{|c|}{ PROIMMSE-IIA-UNAM } \\
\hline Alma Amalia González Cabañas & S. Producción & $\mathrm{D}$ & Sociología \\
\hline Celia Ruiz de Oña Plaza & Desarrollo & $\mathrm{D}$ & Ecología \\
\hline Dolores Camacho Velázquez & Política & M & Economía \\
\hline Fausto Bolom Ton & Ecología & $\mathrm{M}$ & Ecología \\
\hline Gabriel Ascencio Franco & Educación & $\mathrm{D}$ & Antropología \\
\hline Jaime Tomás Page Pliego & Salud pública & $\mathrm{D}$ & Antropología \\
\hline José Federico Morales Barragán & Desarrollo & $\mathrm{D}$ & Economía \\
\hline José Rubén Orantes García & Derecho indígena & D & Antropología \\
\hline Justus Fenner & Historia & $\mathrm{D}$ & Historia \\
\hline Luis Rodríguez Castillo & Política & $\mathrm{D}$ & Antropología \\
\hline Miguel Lisbona Guillén & Historia & $\mathrm{D}$ & Antropología \\
\hline Óscar Sánchez Carrillo & Identidad & $\mathrm{M}$ & Antropología \\
\hline Otto Schumann Gálvez & Lenguas indígenas & $\mathrm{D}$ & Lingüística \\
\hline
\end{tabular}

*Los temas atribuidos son aproximaciones que buscan conformar grupos indicativos; los hice a partir de lo consignado en los directorios de las instituciones de adscripción, y en su ausencia por mis propias apreciaciones. 


\section{Gráfica 1.}

Artículos por revista. Chiapas: 1985-2010

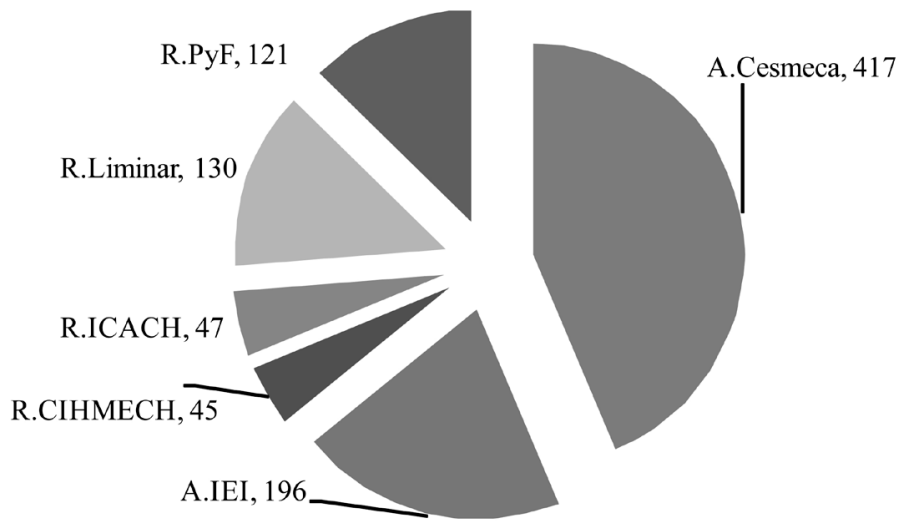

Gráfica 2.

Artículos por año. Chiapas: 1985-2010

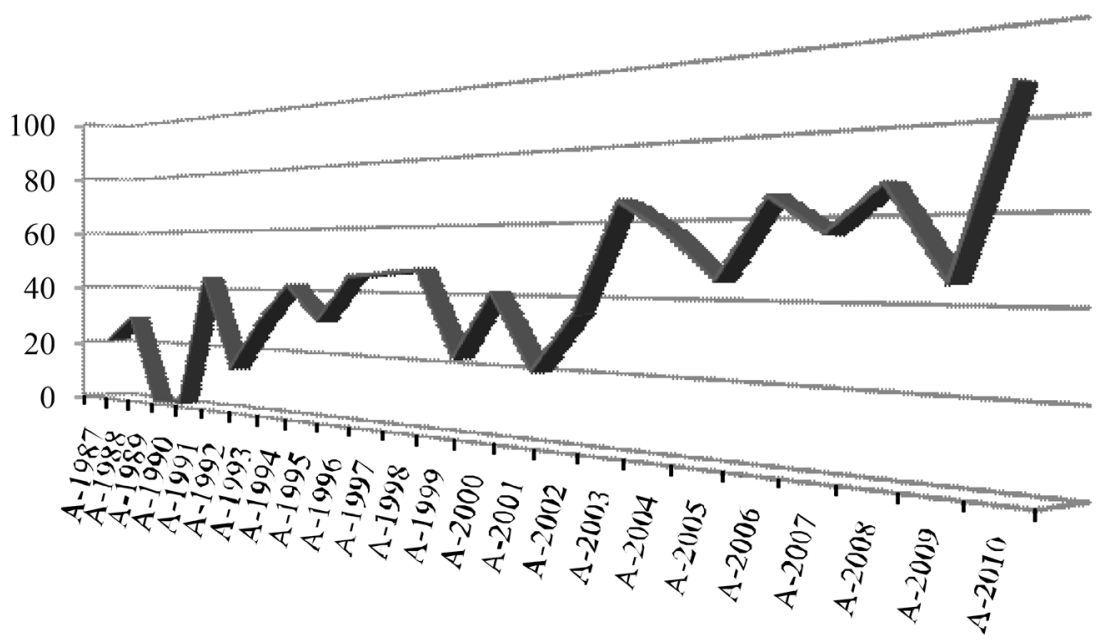




\section{Gráfica 3.}

Artículos por área de conocimiento. Chiapas: 1985-2010

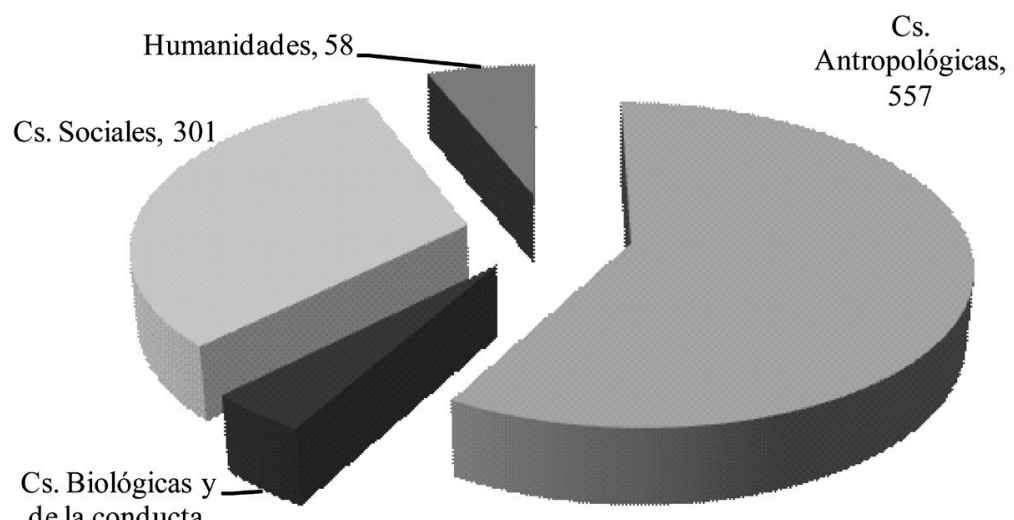

de la conducta,

40

\section{Gráfica 4.}

Artículos por disciplina. Chiapas: 1985-2010

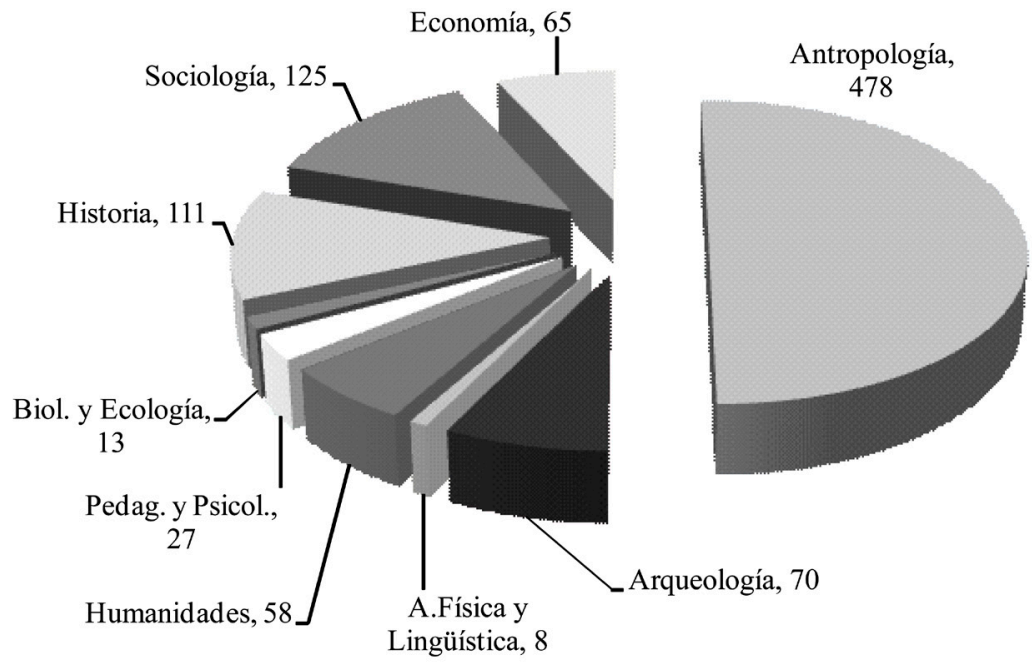

Revista LiminaR. Estudios sociales y humanísticos, año 9, vol. IX, núm. 1, junio de 2011, San Cristóbal de Las Casas, Chiapas, México. ISSN: 1665-8027 


\section{Gráfica 5.}

Artículos por tema. Chiapas: $1985-2010$

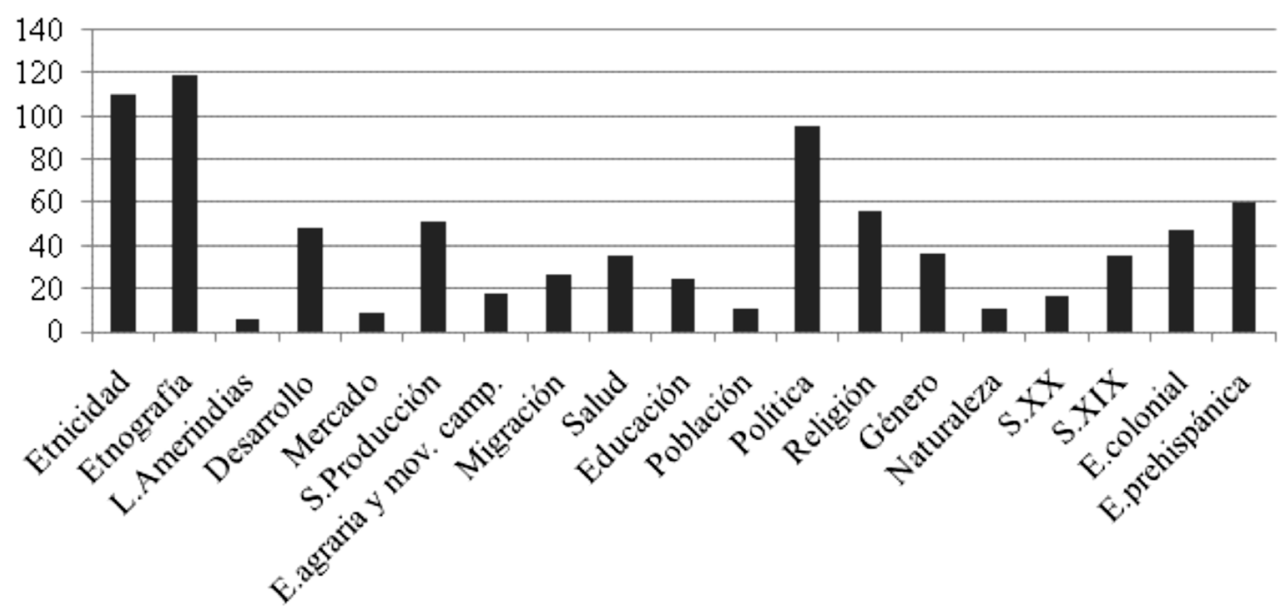

Gráfica 6.

Artículos por escala del estudio. Chiapas: 1985-2010

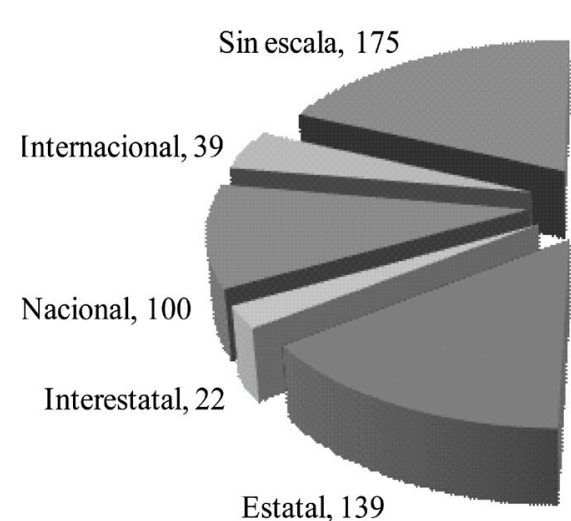

Local, 122

Estatal, 139

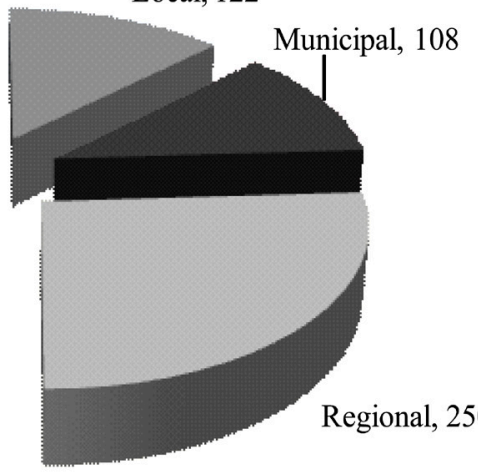




\section{Gráfica 7.}

Artículos por ubicación del estudio. Chiapas: 1985-2010

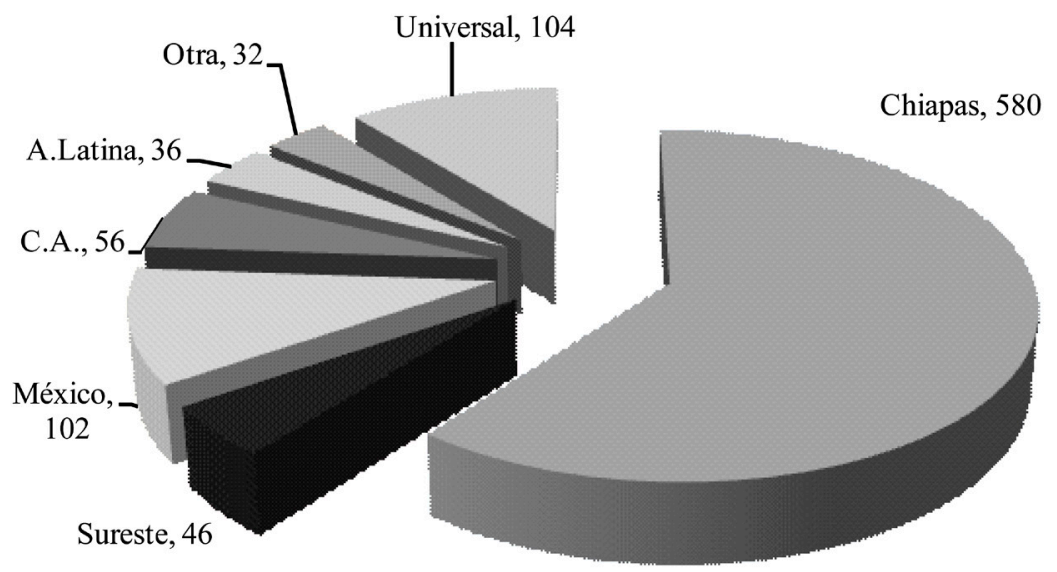

\section{Gráfica 8.}

Artículos por adscripción del autor. Chiapas: 1985-2010

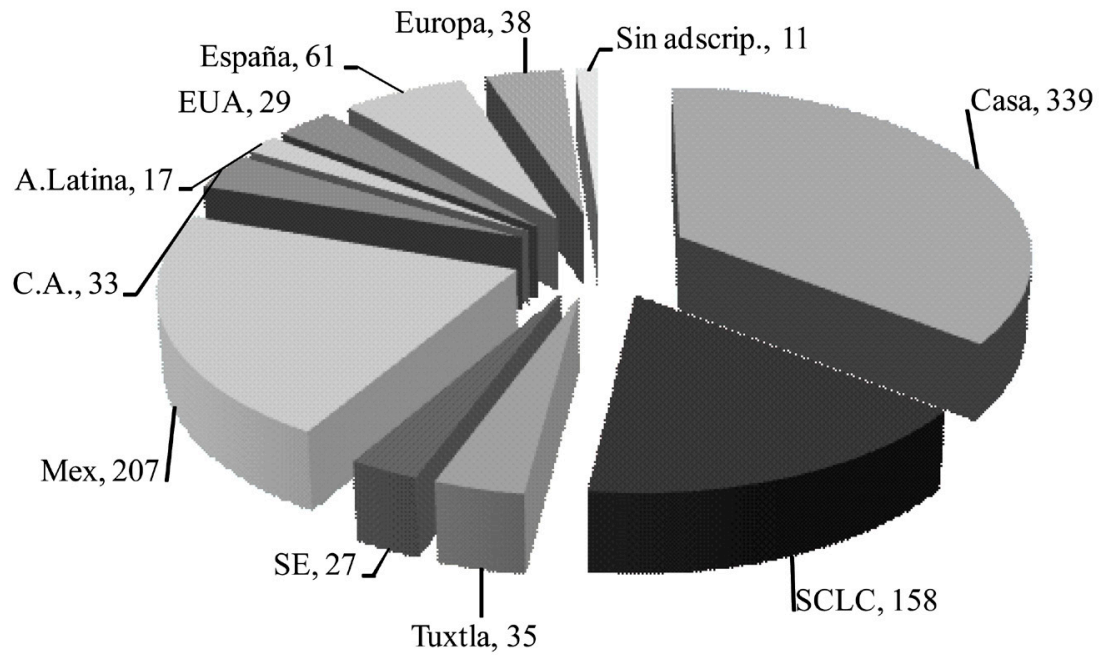




\section{Gráfica 9.}

Artículos en coautoría. Chiapas: 1985-2010.

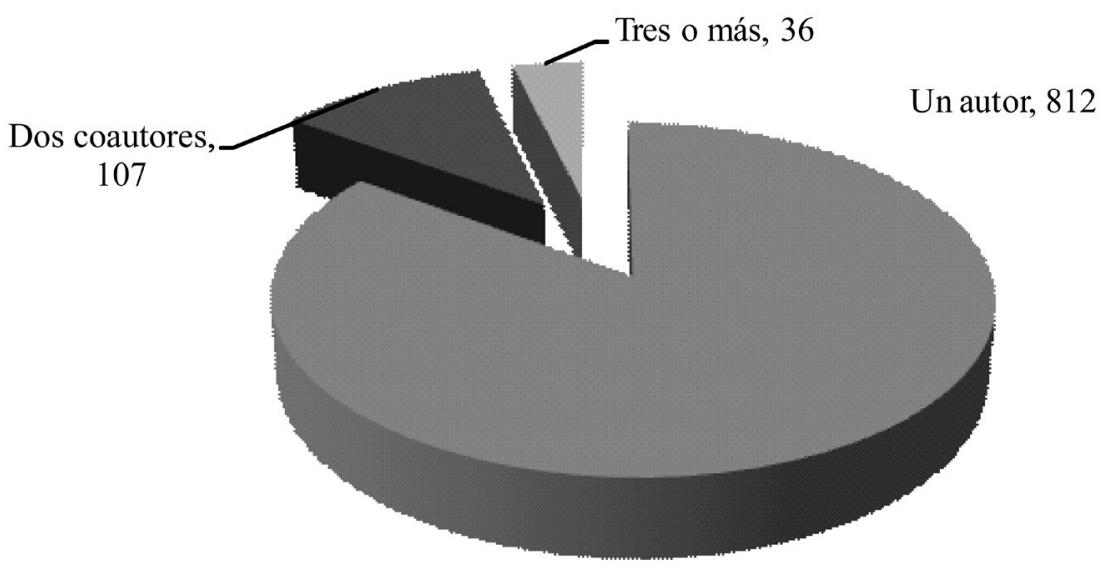

Gráfica 10.

Autores por artículos publicados. Chiapas: 1985-2010.

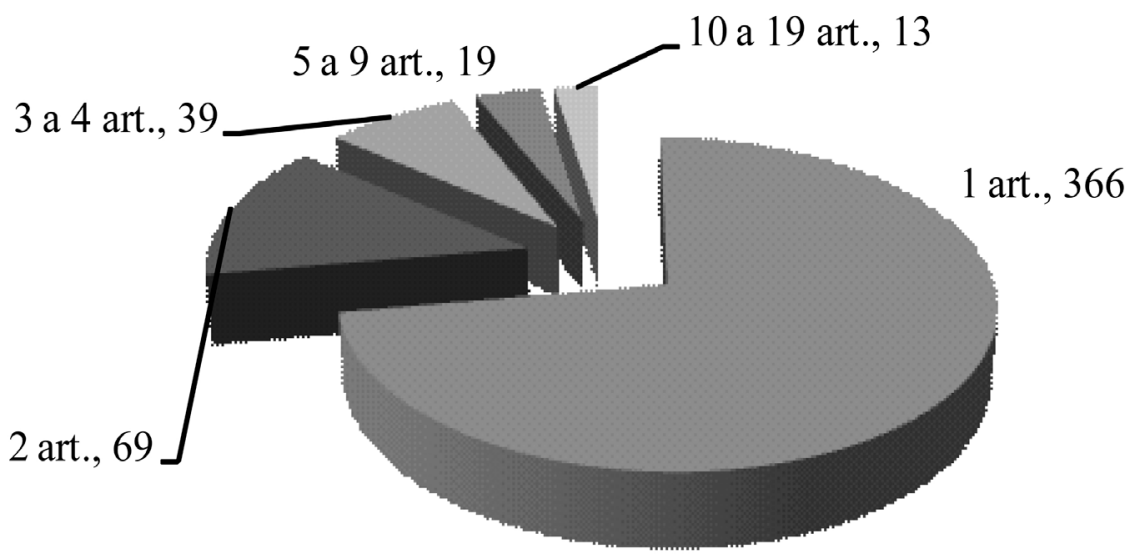

